Curr Opin Rheumatol. 2017 January ; 29(1): 65-70. doi:10.1097/BOR.0000000000000357.

\title{
Disentangling the Role of Neutrophil Extracellular Traps in Rheumatic Diseases
}

\author{
Yaíma L. Lightfoot ${ }^{1}$ and Mariana J. Kaplan ${ }^{1}$ \\ ${ }^{1}$ Systemic Autoimmunity Branch, National Institute of Arthritis and Musculoskeletal and Skin \\ Diseases, National Institutes of Health, Bethesda, Maryland, USA.
}

\begin{abstract}
Purpose of review-A breakdown of immune tolerance to self-antigens in a genetically predisposing background, precipitated by environmental triggers, contributes to the development of systemic autoimmune diseases. Renewed interest in the immunomodulatory capabilities of neutrophils in systemic autoimmunity has identified neutrophil extracellular trap (NET) formation as a distinguishing action of neutrophils in afflicted hosts.
\end{abstract}

Recent findings-Oxidation of nucleic acids and posttranslational modifications of proteins distinctly occur during NET formation and may promote enhanced immunogenicity. Various autoantibodies, immune complexes and other inflammatory stimuli have been recently reported to promote NET formation in individuals with autoimmune diseases. Associations between level of NETosis and adverse outcomes in systemic autoimmune diseases, including thrombosis, adverse pregnancy outcomes and renal disease continue to be investigated.

Summary-Understanding the putative pathogenic role and sequelae of NETosis in rheumatic diseases is a major focus of ongoing research efforts. Mechanisms elucidated by these discoveries may provide novel therapeutic targets to inhibit NET formation and/or promote the clearance of immunogenic netting material.

\section{Keywords}

neutrophil extracellular traps; rheumatic diseases; systemic autoimmunity; thrombosis; glomerulonephritis

\section{Introduction}

Although first described as a mechanism to neutralize and eliminate microorganisms [1], work performed over the last decade has highlighted the putative role neutrophil extracellular traps (NETs) in autoimmunity and inflammatory disorders [2,3]. During the process of NETosis, the integrity of neutrophil intracellular membranes is lost, thereby allowing intermixing of cellular components. Subsequently, a mosaic of granular and

Corresponding Author: Mariana J. Kaplan, M.D., Systemic Autoimmunity Branch, National Institute of Arthritis and Musculoskeletal and Skin Diseases (NIAMS), National Institutes of Health (NIH), 10 Center Drive, 6D/47C, Bethesda, MD, 20892, Phone number: (301) 496-0517, mariana.kaplan@nih.gov. 
cytosolic content, affixed to cellular DNA (of nuclear and/or mitochondrial origin [4]), is extruded from neutrophils. Mechanisms of NET formation in vitro have been described in some detail [5]; nonetheless, the in vivo process remains poorly understood. Histone citrullination, catalyzed by peptidylarginine deiminase 4 (PAD4), appears to be required for NETosis [6,7], at least under specific forms of stimulation, while the exact roles and sources of reactive oxygen species (ROS) are still being defined.

In the context of systemic autoimmune diseases, patient-derived neutrophils undergo spontaneous NETosis more readily, and a number of the molecules externalized as a result, including double-stranded (ds)DNA and myeloperoxidase (MPO), are recognized as autoantigens by the adaptive immune system [8]. Moreover, NET proteins exacerbate inflammatory responses, and NET component-directed autoantibodies and immune complexes potentiate further NET formation, which may then result in a devastating feedforward inflammatory loop in susceptible individuals. In this Review, we discuss the most recent evidence implicating NETs in the pathogenesis of rheumatic diseases, and explore potential therapeutic targets arising from these discoveries.

\section{Environmental Influences on NETosis}

A complex interaction between genetic and environmental factors contributes to the development of autoimmune diseases. Infections, smoking, ultraviolet light, and certain drugs [9-12] encompass some the proposed environmental exposure risks. Of the aforementioned mediators, infection-induced NETosis presents an obvious link between the environment and disease progression. However, other factors linked to autoimmunity are now being recognized as able to promote NET formation. For instance, it was recently reported that nicotine, the major addictive component of tobacco and an environmental factor associated with some systemic autoimmune diseases, binds nicotine acetylcholine receptors on neutrophils and induces NETs in a dose-dependent manner [13]. Albeit delayed, like PMA-induced NET release [14], nicotine stimulated NETs via the activation of Akt and PAD4. Conversely, the process was found to be ROS-independent [13]. In a separate study, ethyl mercury (EtHg) and inorganic mercuric ions $\left(\mathrm{Hg}^{2+}\right)$ also promoted NET formation in vitro [15]. EtHg and $\mathrm{Hg}^{2+}$ stimulation led to the production of NADPH oxidase-independent ROS. Although the source of ROS was postulated to be the mitochondria, it was not formally tested [15]. These studies suggest that the well-recognized contribution of certain environmental factors to the development of autoimmunity may be in part mediated by their ability to stimulate neutrophils to undergo NETosis. In contrast, the differences described in the triggering pathways underscore the need to identify commonalities among the different forms of NETosis if one is to successfully target this process.

\section{NETs in Systemic Lupus Erythematosus (SLE)}

Dysregulated innate and adaptive immune responses contribute to the development of systemic lupus erythematosus (SLE). The presence of a specific subset of low-density granulocytes (LDGs), characterized by enhanced proinflammatory cytokine and type I interferon (IFN) production and heightened NETosis, has been previously described in 
patients with SLE $[16,17]$. Failure to degrade and clear circulating NETs is not only associated with kidney involvement in SLE, but NET-forming neutrophils are also found in the glomeruli of patients with lupus nephritis [17,18]. In addition, NETs were identified in the intervillous space of placentas from lupus patients [19], pointing to NETosis as a probable process contributing to the risk of adverse pregnancy outcomes noted in SLE.

Consistent with the fact that several pathways may lead to NET release, mitochondrial ROS production was recently found to precede spontaneous NETosis by lupus LDGs, as well as NETosis induced by ribonuclear protein-immune complexes (RNP ICs) in normal-density neutrophils [4]. These spontaneous and RNP IC-induced NETs were enriched in oxidized mitochondrial DNA (mtDNA), which potently induced a type I IFN response in a STINGdependent manner [4]. Oxidized mtDNA is interferogenic independent of NETs [20]; however, extrusion of oxidized mtDNA may be significantly hindered by targeting excessive mitochondrial ROS production and/or NETosis. Indeed, in vivo scavenging of mitochondrial ROS in MRL/lpr lupus-prone mice, significantly decreased NETosis, renal immune complex deposition, type I-IFN responses and anti-dsDNA antibody production [4]. Furthermore, disruption of NET formation through PAD inhibition protected MRL/lpr mice from lupusrelated organ involvement, including the vasculature, skin, and kidneys [21] while abrogating NET formation. Amelioration of lupus development in MRL/lpr mice was also achieved through the pharmacologic inhibition of the Janus kinase (JAK)-signal transducer and activator of transcription (STAT) pathway with Tofacitinib [22]. Although the observed therapeutic effects were likely multifaceted, treated mice demonstrated reduced spontaneous and lipopolysaccharide (LPS)-induced NETosis [22]. Lastly, a cohort of SLE patients seemed to benefit from add-on Metformin when compared to standard of care treatment [23]. Neutrophils pretreated with Metformin underwent reduced NETosis and extruded fewer mtDNA copies after PMA stimulation in vitro [23]. While in this study the exact mechanism by which Metformin worked in SLE was not elucidated, previous studies have emphasized the glucose-metabolism requirements of NETosis [24].

\section{NETs in Antiphospholipid Syndrome}

Primary antiphospholipid syndrome (APS) is a heterogeneous autoimmune disease that confers significant risk of spontaneous thrombosis and pregnancy loss. Antiphospholipid antibodies (aPLs) and autoantibodies specific for beta-2 glycoprotein I $\left(\beta_{2} \mathrm{GPI}\right)$ are detected in APS patients. A subset of SLE patients develops an autoimmunity-associated disease, referred to as secondary APS. Of note, both patients with primary and secondary APS demonstrated increased frequencies of circulating LDGs [25], known sources of spontaneous NET formation. Normal density granulocytes from APS patients were also primed to undergo spontaneous NETosis, and patient-derived anti- $\beta_{2}$ GPI-IgG stimulated ROS-dependent NETosis in control neutrophils [26]. Further in vitro investigations showed that aPL-induced NETs had prothrombotic potential [26], thereby identifying NETosis as a possible mechanism for thrombi formation in APS. Still, in order to inhibit NET formation in the context of thrombosis, additional in vitro and in vivo mechanistic studies are needed. For instance, targeting neutrophil elastase (NE), a serine protease implicated in chromatin decondensation during NETosis, did not suppress NETosis in vivo nor did it protect from thrombotic events in and experimental model of deep vein thrombosis [27]. 


\section{NETs in Antineutrophil Cytoplasmic Antibody-Associated Vasculitis}

Antineutrophil cytoplasmic antibody-associated vasculitis (AAV) refers to a subset of smallvessel vasculitides characterized by the presence of antineutrophil cytoplasmic antibodies (ANCAs) [28]. Interestingly, the major target antigens of ANCAs are MPO (MPO-ANCA) and PR3 (PR3-ANCA), two neutrophil granular proteins found within NETs and implicated in NET formation [29,30]. Not surprisingly, the presence of LDGs is also a common feature of patients with AAV [30].

As in SLE, NETosis in AAV is linked with glomerulonephritis [29,31]. Kidney biopsies from patients with MPO-ANCA demonstrated that over $60 \%$ of the patients had NETs within their glomeruli, and of these, a large number had NETs in more than half of the glomeruli visualized, indicating that NETosis is not a rare event in MPO-ANCA glomerulonephritis [31]. The presence of NETs in the kidneys of MPO-ANCA patients were independently found to correlate with MPO-ANCA affinity, but not titer, which may be indicative of differences in the pathogenicity of ANCAs [32]. NETs are also detected in AAV-associated thrombi [33]. The hypercoagulability and enhanced risk for thromboembolic events observed in these patients may now be partly explained by the propensity of C5a-primed neutrophils to form NETs containing tissue factor (TF), the main physiologic initiator of coagulation, in response to ANCA IgG stimulation [34].

Animal models of AAV have been helpful in dissecting the putative role of NETs in the pathogenesis of the disease. A recent study using a mouse model of severe glomerulonephritis showed that in vivo neutralization of extracellular histones protected the animals from the kidney lesions observed in untreated mice, and the protection phenocopied prevention of NETosis by chemical inhibition of PAD activity [35], suggesting that histone extrusion by netting neutrophils contributes to kidney damage. PAD inhibition in a mouse model of MPO-ANCA also demonstrated some therapeutic potential, as the treated mice developed significantly lower MPO-ANCA titers [36]. No kidney involvement was observed in the untreated mice of the latter study; therefore, the effects of pan-PAD activity inhibition on glomerulonephritis could not be measured [36].

As mentioned above, despite the diagnostic use of ANCAs, no consensus has been reached regarding their potential pathogenic role in AAV. It is possible that secondary danger signals or targets must be involved for disease progression. In support of this idea, in vitro stimulation of NETosis by ANCA-positive IgG was enhanced when neutrophils were pretreated with High-mobility group box 1 protein (HMGB1), a nuclear protein recognized as a danger-associated molecular pattern in the context of proinflammatory cell death pathways [37]. Similarly, co-administration of subnephritogenic anti-glomerular basement membrane antibodies and anti-MPO antibodies led to higher renal pathology and intraglomerular NETosis in a rat model of glomerulonephritis [38]. Moreover, recent evidence for a physiologic role of some antibodies with ANCA specificity comes from a report showing that circulating levels of NET remnants (measured as surrogates of NETosis) correlated positively with disease activity, but negatively with ANCAs [39], suggesting that a subset of ANCAs may aid in NET clearance. In contrast, levels of circulating NETs could not distinguish patients in remission from active patients in a different cohort [40]. 
Nonetheless, several studies support the hypothesis that exposure of extracellular MPO and PR3, via aberrant and/or exacerbated NETosis, promote a breakdown in tolerance to these ANCA-targeted antigens.

\section{NETs in Rheumatoid Arthritis}

Rheumatoid arthritis (RA) is a systemic autoimmune disease characterized by synovial joint inflammation and damage. The development of RA evolves from asymptomatic autoimmunity (i.e., the production of pathogenic autoantibodies), to clinically active disease in a process that can take several years [41]. Autoreactive antibodies in RA include autoantibodies to citrullinated antigens (ACPAs) as well as rheumatoid factor (RF). In recent years, NETosis has been implicated in the pathogenesis of RA. Previous studies have shown that, in RA, neutrophils are not only increased in the synovial fluid, but are also more likely to undergo NETosis, thereby externalizing citrullinated autoantigens [42]. Excess NETosis correlates with levels of ACPAs and systemic inflammation in RA patients [42], and may therefore have diagnostic use to identify RA cases [43].

More recently, it was demonstrated that neutrophils undergoing NETosis release enzymatically active PAD isoforms, both diffuse and affixed to NETs [44]. Moreover, ectopic lymphoid structure-resident B cells generate NET-specific, high-affinity ACPAs [45], suggesting a self-perpetuating cycle, whereby NETosis not only exposes citrullinated autoantigens, but also promotes the citrullination of antigens in the synovium, and this, in turn, results in heightened autoimmunoreactivity and affinity maturation of synovial B cells. Importantly, a single-nucleotide polymorphism, which converts an arginine (R620) to a tryptophan (W620) in PTPN22 and confers the highest risk of RA development outside of the HLA region, was revealed to disrupt regulatory PTPN22-PAD4 interactions, resulting in enhanced protein citrullination and spontaneous NETosis [46]. Taken together, these data provide compelling evidence incriminating NETosis in the development of RA in at-risk individuals. It will be important to establish whether enhanced NETosis associated to externalized citrullinated autoantigens also occurs at pre-clinical and clinical stages in mucosal sites where the initial pathogenic pathways in RA have been proposed to take place [47]. Notwithstanding, the rate of RA progression suggests additional predispositions/risk factors are likely necessary to achieve clinical manifestations of the disease.

\section{Therapeutic Targets in NETosis}

Given the persistence of NETs in autoimmunity, disrupting these lattices with exogenous molecules or drugs may be of therapeutic value for patients suffering from systemic autoimmune diseases. As an example, DNases, required not only for the disassembly of NETs [18], but also for the digestion of immunogenic apoptotic cell microparticles [48], may contribute to the clearance of pre-existing NETs. Similarly, PAD inhibitors continue to show promise in the prevention of NETosis in pre-clinical settings $[21,36]$. Specific inhibition of mitochondrial ROS-induced NETosis [4], through the use of mitochondrial ROS scavengers, may help ameliorate clinical symptoms. The JAK/STAT pathway may also contribute to neutrophil activation and NETosis; its targeting reduced NETosis in vivo [22], but the mechanism warrants further investigation. In addition, improving our understanding 
on the mechanisms of action of currently used rheumatologic drugs with regards to their modulation of NETosis and other neutrophil functions is warranted. Lastly, neutrophils depend on glycolysis for their energy; therefore, molecules that target the glycolytic pathway may also prevent NETosis. Nonetheless, a better understanding of the immunometabolism of NETosis is needed [49]. Importantly, it will be very important to further our understanding on how selectively targeting NETosis could affect host responses to microbes. Identification neutrophil subsets with distinct pathogenic characteristics, such as LDGs, may promote targeted therapies while preserving crucial aspects of neutrophil-mediated host defense.

\section{Concluding Remarks}

The observation that many systemic autoimmune diseases are not only associated with heightened NETosis and impaired NET clearance, but also show a greater frequency of adverse events correlating with NETosis, strongly suggests that NETs contribute to the pathogenesis of these diseases (Table 1). Whether NETosis is a triggering event or has an additive effect to other factors, such as the environment or a predisposing genetic background, remains to be fully addressed. Additionally, a more detailed mechanistic understanding of NETosis in a genetically susceptible background may allow for the specific inhibition of autoimmunity-promoting NETosis while preserving protective NET formation. To this end, investigating whether mitochondrial ROS-induced NETosis and oxidation of mtDNA (as seen in SLE [4]) are pathogenic processes spanning other systemic autoimmune diseases, particularly those associated to enhanced type I IFN responses, may prove to be useful in designing novel therapeutic approaches.

\section{Acknowledgments}

None

Financial support: This work was supported by the Intramural Research Program at NIAMS/NIH (ZIAAR041199).

\section{References}

1. Brinkmann V, Reichard U, Goosmann C, Fauler B, Uhlemann Y, Weiss DS, Weinrauch Y, Zychlinsky A. Neutrophil extracellular traps kill bacteria. Science. 2004; 303:1532-1535. [PubMed: 15001782]

2. Kaplan MJ, Radic M. Neutrophil extracellular traps: double-edged swords of innate immunity. J Immunol. 2012; 189:2689-2695. [PubMed: 22956760]

3. Sørensen OE, Borregaard N. Neutrophil extracellular traps - the dark side of neutrophils. J Clin Invest. 2016; 126:1612-1620. [PubMed: 27135878]

**4. Lood C, Blanco LP, Purmalek MM, Carmona-Rivera C, De Ravin SS, Smith CK, Malech HL, Ledbetter JA, Elkon KB, Kaplan MJ. Neutrophil extracellular traps enriched in oxidized mitochondrial DNA are interferogenic and contribute to lupus-like disease. Nat Med. 2016; 22:146-153. Oxidized mtDNA is externalized by NETs derived from lupus neutrophils or in NETs induced by RNP-ICs, leading to a STING_dependent interferogenic response that may be relevant to disease pathogenesis. Mitochondria were identified as a major source of ROS production during NETosis, both in lupus patients, and in individuals with chronic granulomatous disease. This study suggests that mitochondrial ROS are important drivers of immunogenicity in SLE. [PubMed: 26779811]

5. Brinkmann V, Zychlinsky A. Neutrophil extracellular traps: is immunity the second function of chromatin? J Cell Biol. 2012; 198:773-783. [PubMed: 22945932] 
6. Wang Y, Li M, Stadler S, Correll S, Li P, Wang D, Hayama R, Leonelli L, Han H, Grigoryev SA, et al. Histone hypercitrullination mediates chromatin decondensation and neutrophil extracellular trap formation. J Cell Biol. 2009; 184:205-213. [PubMed: 19153223]

7. Li P, Li M, Lindberg MR, Kennett MJ, Xiong N, Wang Y. PAD4 is essential for antibacterial innate immunity mediated by neutrophil extracellular traps. J Exp Med. 2010; 207:1853-1862. [PubMed: 20733033]

8. Grayson PC, Kaplan MJ. At the Bench: Neutrophil extracellular traps (NETs) highlight novel aspects of innate immune system involvement in autoimmune diseases. J Leukoc Biol. 2016; 99:253-264. [PubMed: 26432901]

9. Bogdanos DP, Smyk DS, Rigopoulou EI, Sakkas LI, Shoenfeld Y. Infectomics and autoinfectomics: a tool to study infectious-induced autoimmunity. Lupus. 2015; 24:364-373. [PubMed: 25801879]

**10. Nielsen PR, Kragstrup TW, Deleuran BW, Benros ME. Infections as risk factor for autoimmune diseases - A nationwide study. J Autoimmun. 2016 This study followed a large Danish cohort and found that infections are a significant risk factor for the development of autoimmunity. The association included 29 autoimmune diseases.

11. Perricone C, Versini M, Ben-Ami D, Gertel S, Watad A, Segel MJ, Ceccarelli F, Conti F, Cantarini L, Bogdanos DP, et al. Smoke and autoimmunity: The fire behind the disease. Autoimmun Rev. 2016; 15:354-374. [PubMed: 26772647]

12. Cañas CA, Cañas F, Bonilla-Abadía F, Ospina FE, Tobón GJ. Epigenetics changes associated to environmental triggers in autoimmunity. Autoimmunity. 2016; 49:1-11. [PubMed: 26369426]

13. Hosseinzadeh A, Thompson PR, Segal BH, Urban CF. Nicotine induces neutrophil extracellular traps. J Leukoc Biol. 2016

14. Douda DN, Yip L, Khan MA, Grasemann H, Palaniyar N. Akt is essential to induce NADPHdependent NETosis and to switch the neutrophil death to apoptosis. Blood. 2014; 123:597-600. [PubMed: 24458280]

15. Haase H, Hebel S, Engelhardt G, Rink L. Ethylmercury and $\mathrm{Hg} 2+$ induce the formation of neutrophil extracellular traps (NETs) by human neutrophil granulocytes. Arch Toxicol. 2016; 90:543-550. [PubMed: 25701957]

16. Denny MF, Yalavarthi S, Zhao W, Thacker SG, Anderson M, Sandy AR, McCune WJ, Kaplan MJ. A distinct subset of proinflammatory neutrophils isolated from patients with systemic lupus erythematosus induces vascular damage and synthesizes type I IFNs. J Immunol. 2010; 184:32843297. [PubMed: 20164424]

17. Villanueva E, Yalavarthi S, Berthier CC, Hodgin JB, Khandpur R, Lin AM, Rubin CJ, Zhao W, Olsen SH, Klinker M, et al. Netting neutrophils induce endothelial damage, infiltrate tissues, and expose immunostimulatory molecules in systemic lupus erythematosus. J Immunol. 2011; 187:538-552. [PubMed: 21613614]

18. Hakkim A, Fürnrohr BG, Amann K, Laube B, Abed UA, Brinkmann V, Herrmann M, Voll RE, Zychlinsky A. Impairment of neutrophil extracellular trap degradation is associated with lupus nephritis. Proc Natl Acad Sci U S A. 2010; 107:9813-9818. [PubMed: 20439745]

**19. Marder W, Knight JS, Kaplan MJ, Somers EC, Zhang X, O'Dell AA, Padmanabhan V, Lieberman RW. Placental histology and neutrophil extracellular traps in lupus and pre-eclampsia pregnancies. Lupus Sci Med. 2016; 3:e000134. NETs were found in the placental intervillous space of patients with pre-eclampsia (with or without lupus) as well as in lupus patients without pre-eclampsia. NETs may contribute to the negative pregnancy outcomes associated with these conditions. [PubMed: 27158525]

20. Caielli S, Athale S, Domic B, Murat E, Chandra M, Banchereau R, Baisch J, Phelps K, Clayton S, Gong $\mathrm{M}$, et al. Oxidized mitochondrial nucleoids released by neutrophils drive type I interferon production in human lupus. J Exp Med. 2016; 213:697-713. [PubMed: 27091841]

*21. Knight JS, Subramanian V, O'Dell AA, Yalavarthi S, Zhao W, Smith CK, Hodgin JB, Thompson PR, Kaplan MJ. Peptidylarginine deiminase inhibition disrupts NET formation and protects against kidney, skin and vascular disease in lupus-prone MRL/lpr mice. Ann Rheum Dis. 2015; 74:2199-2206. This work provides proof-of-concept that PAD inhibition may represent a therapeutic target in SLE, at least in part, through NET inhibition. [PubMed: 25104775] 
**22. Furumoto Y, Smith CK, Blanco L, Zhao W, Brooks SR, Thacker SG, Zarzour A, Sciumè G, Tsai WL, Trier AM, et al. Tofacitinib ameliorates murine lupus and its associated vascular dysfunction. Arthritis Rheumatol. 2016 Disease measurements, including autoantibody and proinflammatory cytokine production, interferon responses, NET formation, skin inflammation, vascular endothelial function, and nephritis, were significantly improved by treatment with the Jak inhibitor Tofacitinib in a lupus experimental model.

23. Wang H, Li T, Chen S, Gu Y, Ye S. Neutrophil Extracellular Trap Mitochondrial DNA and Its Autoantibody in Systemic Lupus Erythematosus and a Proof-of-Concept Trial of Metformin. Arthritis Rheumatol. 2015; 67:3190-3200. [PubMed: 26245802]

24. Rodríguez-Espinosa O, Rojas-Espinosa O, Moreno-Altamirano MM, López-Villegas EO, SánchezGarcía FJ. Metabolic requirements for neutrophil extracellular traps formation. Immunology. 2015; 145:213-224. [PubMed: 25545227]

25. van den Hoogen LL, Fritsch-Stork RD, van Roon JA, Radstake TR. Low-Density Granulocytes Are Increased in Antiphospholipid Syndrome and Are Associated With Anti- $\beta 2$-Glycoprotein I Antibodies: Comment on the Article by Yalavarthi et al. Arthritis Rheumatol. 2016; 68:13201321. [PubMed: 26749538]

**26. Yalavarthi S, Gould TJ, Rao AN, Mazza LF, Morris AE, Núñez-Álvarez C, Hernández-Ramírez D, Bockenstedt PL, Liaw PC, Cabral AR, et al. Release of neutrophil extracellular traps by neutrophils stimulated with antiphospholipid antibodies: a newly identified mechanism of thrombosis in the antiphospholipid syndrome. Arthritis Rheumatol. 2015; 67:2990-3003. This study provides the first evidence implicating NETosis as a prothrombotic driver in antiphospholipid syndrome, with potentially important therapeutic implications. [PubMed: 26097119]

27. Martinod K, Witsch T, Farley K, Gallant M, Remold-O'Donnell E, Wagner DD. Neutrophil elastase-deficient mice form neutrophil extracellular traps in an experimental model of deep vein thrombosis. J Thromb Haemost. 2016; 14:551-558. [PubMed: 26712312]

28. Jarrot PA, Kaplanski G. Pathogenesis of ANCA-associated vasculitis: An update. Autoimmun Rev. 2016; 15:704-713. [PubMed: 26970490]

29. Kessenbrock K, Krumbholz M, Schönermarck U, Back W, Gross WL, Werb Z, Gröne HJ, Brinkmann V, Jenne DE. Netting neutrophils in autoimmune small-vessel vasculitis. Nat Med. 2009; 15:623-625. [PubMed: 19448636]

*30. Grayson PC, Carmona-Rivera C, Xu L, Lim N, Gao Z, Asare AL, Specks U, Stone JH, Seo P, Spiera RF, et al. Neutrophil-Related Gene Expression and Low-Density Granulocytes Associated With Disease Activity and Response to Treatment in Antineutrophil Cytoplasmic AntibodyAssociated Vasculitis. Arthritis Rheumatol. 2015; 67:1922-1932. A granulocyte gene signature in whole blood correlated with differential responses to treatment in AAV. The gene signature was confirmed in PBMCs, leading to the characterization of a low-density granulocyte population in AAV patients that is prone to undergo enhanced NET formation. [PubMed: 25891759]

31. O'Sullivan KM, Lo CY, Summers SA, Elgass KD, McMillan PJ, Longano A, Ford SL, Gan PY, Kerr PG, Kitching AR, et al. Renal participation of myeloperoxidase in antineutrophil cytoplasmic antibody (ANCA)-associated glomerulonephritis. Kidney Int. 2015; 88:1030-1046. [PubMed: 26176828]

32. Yoshida M, Yamada M, Sudo Y, Kojima T, Tomiyasu T, Yoshikawa N, Oda T. Myeloperoxidase anti-neutrophil cytoplasmic antibody affinity is associated with the formation of neutrophil extracellular traps in the kidney and vasculitis activity in myeloperoxidase anti-neutrophil cytoplasmic antibody-associated microscopic polyangiitis. Nephrology (Carlton). 2016; 21:624629. [PubMed: 26833773]

33. Nakazawa D, Tomaru U, Yamamoto C, Jodo S, Ishizu A. Abundant neutrophil extracellular traps in thrombus of patient with microscopic polyangiitis. Front Immunol. 2012; 3:333. [PubMed: 23162551]

**34. Huang YM, Wang H, Wang C, Chen M, Zhao MH. Promotion of hypercoagulability in antineutrophil cytoplasmic antibody-associated vasculitis by $\mathrm{C} 5 \mathrm{a}$-induced tissue factorexpressing microparticles and neutrophil extracellular traps. Arthritis Rheumatol. 2015; 67:2780- 
2790. This study proposes a mechanism by which NETs, microparticles and complement promote thrombosis in AAV. [PubMed: 26097236]

*35. Kumar SV, Kulkarni OP, Mulay SR, Darisipudi MN, Romoli S, Thomasova D, Scherbaum CR, Hohenstein B, Hugo C, Müller S, et al. Neutrophil Extracellular Trap-Related Extracellular Histones Cause Vascular Necrosis in Severe GN. J Am Soc Nephrol. 2015; 26:2399-2413. An experimental model of glomerulonephritis was used to confirm that histone release during NETosis is cytotoxic and induces inflammatory responses. [PubMed: 25644111]

36. Kusunoki Y, Nakazawa D, Shida H, Hattanda F, Miyoshi A, Masuda S, Nishio S, Tomaru U, Atsumi T, Ishizu A. Peptidylarginine Deiminase Inhibitor Suppresses Neutrophil Extracellular Trap Formation and MPO-ANCA Production. Front Immunol. 2016; 7:227. [PubMed: 27375623]

37. Ma YH, Ma TT, Wang C, Wang H, Chang DY, Chen M, Zhao MH. High-mobility group box 1 potentiates antineutrophil cytoplasmic antibody-inducing neutrophil extracellular traps formation. Arthritis Res Ther. 2016; 18:2. [PubMed: 26739852]

38. Kanzaki G, Nagasaka S, Higo S, Kajimoto Y, Kanemitsu T, Aoki M, Nagahama K, Natori Y, Tsuboi N, Yokoo T, et al. Impact of anti-glomerular basement membrane antibodies and glomerular neutrophil activation on glomerulonephritis in experimental myeloperoxidaseantineutrophil cytoplasmic antibody vasculitis. Nephrol Dial Transplant. 2016; 31:574-585. [PubMed: 26582929]

39. Söderberg D, Kurz T, Motamedi A, Hellmark T, Eriksson P, Segelmark M. Increased levels of neutrophil extracellular trap remnants in the circulation of patients with small vessel vasculitis, but an inverse correlation to anti-neutrophil cytoplasmic antibodies during remission. Rheumatology (Oxford). 2015; 54:2085-2094. [PubMed: 26170375]

40. Wang H, Sha LL, Ma TT, Zhang LX, Chen M, Zhao MH. Circulating Level of Neutrophil Extracellular Traps Is Not a Useful Biomarker for Assessing Disease Activity in Antineutrophil Cytoplasmic Antibody-Associated Vasculitis. PLoS One. 2016; 11:e0148197. [PubMed: 26840412]

41. Deane KD. Preclinical rheumatoid arthritis (autoantibodies): an updated review. Curr Rheumatol Rep. 2014; 16:419. [PubMed: 24643396]

42. Khandpur R, Carmona-Rivera C, Vivekanandan-Giri A, Gizinski A, Yalavarthi S, Knight JS, Friday S, Li S, Patel RM, Subramanian V, et al. NETs are a source of citrullinated autoantigens and stimulate inflammatory responses in rheumatoid arthritis. Sci Transl Med. 2013; 5:178ra140.

43. Sur Chowdhury C, Giaglis S, Walker UA, Buser A, Hahn S, Hasler P. Enhanced neutrophil extracellular trap generation in rheumatoid arthritis: analysis of underlying signal transduction pathways and potential diagnostic utility. Arthritis Res Ther. 2014; 16:R122. [PubMed: 24928093]

**44. Spengler J, Lugonja B, Ytterberg AJ, Zubarev RA, Creese AJ, Pearson MJ, Grant MM, Milward M, Lundberg K, Buckley CD, et al. Release of Active Peptidyl Arginine Deiminases by Neutrophils Can Explain Production of Extracellular Citrullinated Autoantigens in Rheumatoid Arthritis Synovial Fluid. Arthritis Rheumatol. 2015; 67:3135-3145. ETosis and PAD activity were confirmed to be enhanced in the synovium of RA patients. This study is the first to show that netting neutrophils excrete freely diffused, but enzymatically active, PADs in RA, potentially leading to the citrullination of extracellular antigens. [PubMed: 26245941]

**45. Corsiero E, Bombardieri M, Carlotti E, Pratesi F, Robinson W, Migliorini P, Pitzalis C. Single cell cloning and recombinant monoclonal antibodies generation from RA synovial B cells reveal frequent targeting of citrullinated histones of NETs. Ann Rheum Dis. 2015 This study demonstrated RA synovium-localized immune responses leading to the generation of highaffinity, anti-NET antibodies.

**46. Chang HH, Dwivedi N, Nicholas AP, Ho IC. The W620 Polymorphism in PTPN22 Disrupts Its Interaction With Peptidylarginine Deiminase Type 4 and Enhances Citrullination and NETosis. Arthritis Rheumatol. 2015; 67:2323-2334. A novel link between a genetic risk factor and NETosis was provided. The risk SNP allowed for increased PAD4 activity, which led to enhanced protein citrullination and spontaneous NET formation. [PubMed: 26019128]

47. Catrina AI, Deane KD, Scher JU. Gene, environment, microbiome and mucosal immune tolerance in rheumatoid arthritis. Rheumatology (Oxford). 2016; 55:391-402. [PubMed: 25539828] 
48. Sisirak V, Sally B, D'Agati V, Martinez-Ortiz W, Özçakar ZB, David J, Rashidfarrokhi A, Yeste A, Panea C, Chida AS, et al. Digestion of Chromatin in Apoptotic Cell Microparticles Prevents Autoimmunity. Cell. 2016; 166:88-101. [PubMed: 27293190]

49. O'Neill LA, Kishton RJ, Rathmell J. A guide to immunometabolism for immunologists. Nat Rev Immunol. 2016

50. Kahlenberg JM, Carmona-Rivera C, Smith CK, Kaplan MJ. Neutrophil extracellular trapassociated protein activation of the NLRP3 inflammasome is enhanced in lupus macrophages. J Immunol. 2013; 190:1217-1226. [PubMed: 23267025]

51. Gan PY, Tan DS, Ooi JD, Alikhan MA, Kitching AR, Holdsworth SR. Myeloperoxidase PeptideBased Nasal Tolerance in Experimental ANCA-Associated GN. J Am Soc Nephrol. 2016; 27:385391. [PubMed: 26047792] 


\section{Key Points}

- Heightened NET formation and dysfunctional NET clearance are common features of systemic autoimmune diseases.

- Environmental and genetic risk factors are associated with enhanced NETosis.

- Oxidation of nucleic acids during NETosis may be an important immunogenic factor in systemic rheumatic diseases.

- $\quad$ Blocking NETosis may improve disease outcomes in systemic autoimmunity patients. 


\section{Table 1}

Recent studies implicating NETs in the pathogenesis of systemic autoimmune diseases.

\begin{tabular}{|c|c|c|}
\hline Factor & Finding & Relevant References \\
\hline Genetic Risk & W620 polymorphism in PTPN22 promotes NETs & {$[46]$} \\
\hline Environment & Microorganisms, nicotine, heavy metals induce NETs & {$[2,13,15]$} \\
\hline Innate Immune Responses & $\begin{array}{c}\text { NETS promote activation of the NLRP3 Inflammasome and STING } \\
\text { pathway }\end{array}$ & {$[4,50]$} \\
\hline Adaptive Immune Responses & $\begin{array}{c}\text { NETs promote Autoantibody production, while certain } \\
\text { autoantibodies and immune complexes trigger NETosis }\end{array}$ & $\begin{array}{c}\text { Nets are implicated in vascular damage, thrombosis, } \\
\text { glomerulonephritis, skin disease, and poor pregnancy outcomes }\end{array}$ \\
\hline Organ Damage & & {$[4,16,17,19,21,22,26,31-33,35,38,51]$} \\
\hline
\end{tabular}

